\title{
A Composite Wavelets and Morphology Approach for ECG Noise Filtering
}

\author{
Vikrant Bhateja ${ }^{1}$, Shabana Urooj ${ }^{2}$, Rini Mehrotra ${ }^{1}$, Rishendra Verma ${ }^{1}$, \\ Aimé Lay-Ekuakille ${ }^{3}$, and Vijay Deepak Verma ${ }^{4}$ \\ ${ }^{1}$ Department of Electronics and Communication Engineering, Shri Ramswaroop Memorial \\ Group of Professional Colleges (SRMGPC), Lucknow (U.P.), India \\ ${ }^{2}$ Department of Electrical Engineering, School of Engineering, Gautam Buddha University, \\ Greater-Noida (U.P.), India \\ ${ }^{3}$ Department of Innovation Engineering, University of Salento, Lecce, Italy \\ ${ }^{4}$ Medical University \& Associated Hospitals Greater Noida UP India \\ \{bhateja.vikrant, rini684.mehrotra, verma.rishendra90, \\ shabanabilal\}@gmail.com
}

\begin{abstract}
Noisy ECG signals contain variations in the amplitudes or in the time intervals which represents the abnormalities associated with the heart; thereby making visual diagnosis difficult for cardiovascular diseases. Hence, to facilitate proper analysis of ECG; this paper presents a combination of wavelets analysis and morphological filtering as an approach for noise removal in ECG signals. The proposed algorithm involves sub-band decomposition of ECG signal using bi-orthogonal wavelet family. The wavelet detail coefficients containing the noisy components are then processed by morphological operators using linear structuring elements. The morphological filter processes only the corrupted bands without affecting the signal parameters. Simulation results of the proposed algorithm show noteworthy suppression of noise in terms of higher signal-to-noise ratio preserving the ST segment and R wave of ECG.
\end{abstract}

Index Terms: bi-orthogonal wavelets, detail coefficients, morphological filtering.

\section{Introduction}

Electrocardiogram (ECG) is measure of the voltage fluctuations occurring inside heart because of the potential changes across the cell membrane. During acquisition from the patient's body, ECG signal gets distorted by different types of noise artifacts that can be within a frequency band of interest. ECG signals are plagued by impulse noise, due to muscle activities; it appears as false positive and negative voltage fluctuations (in the form of spikes of short duration) in the ECG signal [1-2]. Therefore, ECG signal conditioning for providing noise removal is a necessary requirement for computer-aided analysis such as QRS detection and temporal alignment. Computer based diagnosis have been demonstrated fertile for various other diseases like breast cancers 
[3]-[6], brain tumors [7]-[8] as well as for diseases based on accumulation of fluid [9]-[12]. In this regard, the reliability issues of the computer-based analysis models reported in former works are of great importance. Numerous variants of morphological filtering operations have been proposed in literature for noise filtering of ECG signals. Processing of ECG signals with different variants of morphological operators employed flat structuring elements were able to reduce ST segment distortion only up to 50\% [13]-[15]. Wavelet based techniques [16]-[18] for ECG noise suppression requires numerous experiments for ruling out scales and thresholds. These algorithms were complex incorporating mother wavelet selection and may require more computation time. R. Verma et al. [19]-[21] proposed another method for impulse noise suppression and baseline correction in ECG employing three as well as two dimensional structuring elements. In the present work, an improved filtering technique using a combination of wavelets and morphology is employed for achieving low distortion and high noise suppression. The rest of this paper is organized as follows. The proposed ECG filtering approach is described in Section 2. Section 3 provides the obtained simulation results and discusses them while the work is concluded under Section 4.

\section{Proposed ECG Filtering Approach}

ECG signal being a non-stationary signal makes it difficult for denoising. A proficient technique for such a non-stationary signal processing is the wavelet transform. The wavelet transform can be used for decomposition of a signal in the time frequency scale plane. Morphological filtering is a non-linear transformation technique primarily used for local modification of geometrical features of a signal. This shape information (of features) is extracted by using a structuring element (of appropriate dimensions) to operate on the input signal. Erosion, dilation, opening and closing are the common morphological operators used. [22]-[23]. An important aspect in noise suppression is the selection of optimum size of the structuring element. Improper selection of structuring element may distort the adjacent wave in the ECG signal. Moreover, the ECG noise filtering by pure morphological filter leaves residual impulse noise which brings variations in the amplitude and time interval of the processed ECG signal. However, biorthogonal wavelet provides frequency domain approach for noise filtering. It is noteworthy that decomposition of ECG signal into detail sub-bands helps in dealing with the residual noise which cannot be removed through morphological filter. Hence, in this work, combination of wavelet and morphology is used for noise filtering of ECG signal. The signal is first decomposed into subbands; the wavelet detail coefficients are then processed by applying morphological operations using linear structuring element. The proposed algorithm comprises of following steps:

Step-1: The algorithm initiates with the decomposition of the ECG signal into subbands. This is done by choice of appropriate wavelet family like Bi-orthogonal wavelets for ECG signal processing. Most of the impulse noise which is characterized by 
high spikes resides in the detail sub-bands. Thus, the detail sub-bands with high frequency spikes are separated for application of proposed morphological filter.

Step-2: The morphological filter involves the application of combination of erosion and dilation operators on the affected sub-bands. ECG signal (A) is mainly corrupted by impulses (or spikes) i.e. very large positive or negative values of very short interval. The noise suppression with the proposed morphological filter involves dilation followed by erosion in the first stage and erosion followed by dilation in second stage using linear structuring element (B). The structuring element should be comparable to the width of impulse noise. The linear structuring element B used in this work consists of an array of ones of size 10. This enables removal of residual impulse noise without introducing any distortion in ECG waveform or loss of information. The results of both the stages are averaged as given by equation (1) to get the denoised detail sub-bands.

$$
\begin{aligned}
& \mathrm{B}=\left[\begin{array}{llllllllll}
1 & 1 & 1 & 1 & 1 & 1 & 1 & 1 & 1 & 1
\end{array}\right] \\
& f=\frac{((\mathrm{A} \theta \mathrm{B}) \oplus \mathrm{B})+((\mathrm{A} \oplus \mathrm{B}) \theta \mathrm{B})}{2}
\end{aligned}
$$

Where: $\mathrm{B}$ is a structuring element, $f$ represents the reconstructed ECG signal, $\theta$ represents erosion operation, $\oplus$ represents dilation operation.

Step-3: The denoised output ECG signal is finally reconstructed from the the processed detail sub-bands through wavelet reconstruction.

\section{$3 \quad$ Results and Discussions}

For simulation purposes in the present work, ECG signals are adapted from internationally accepted MIT-BIH database that consists of recordings which are observed in clinical practice. The ECG recordings were created using two clean recordings (118 and 119) from the MIT-BIH Arrhythmia Database, to which calibrated amounts of noise from record 'em' were added which are termed as Noise Stress Databases [24]. ECG signal interpretation is based on the wave amplitude, time interval and the shape of QRS complexes. These signal parameters are affected by the noise which interfere with the actual information and thereby makes visual diagnosis difficult [25]. It can be noticed that in the fig. 1(a) and 2(a) the QRS complex is corrupted from noise artifacts. The corrupted ECG signal is first decomposed into sub-bands by choice of appropriate wavelets. The noise content in the detail sub-bands is then suppressed by using morphological filtering. The choice of optimal structuring element is experimentally determined by using the Signal to Noise Ratio (SNR) as performance measure for quality evaluation [26]-[27]. It can be seen from fig. 1(b) and 2(b) that impulse noise is reduced by the proposed approach. Evaluation of SNR values will act as tool for the comparison of different families. The SNR value of noisy signals $118 \mathrm{e} 24$, $119 \mathrm{e} 24$ and PTB s0010 were found to be $25.21 \mathrm{~dB}, 36.27 \mathrm{~dB}$ and $24.74 \mathrm{~dB}$ respectively. Upon denoising with the proposed approach, SNR values get enhanced to 32.2 $\mathrm{dB}, 44.5 \mathrm{~dB}$ and $26.5 \mathrm{~dB}$ respectively. 


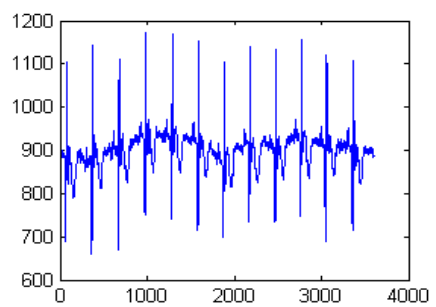

(a)

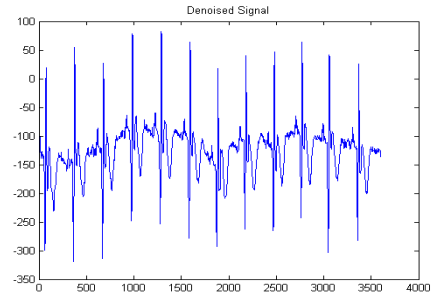

(b)

Fig. 1. (a) Noisy ECG Signal (MIT BIH record 118e24), (b) Denoised ECG Signal using proposed approach

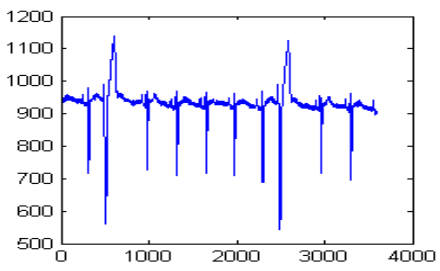

(a)

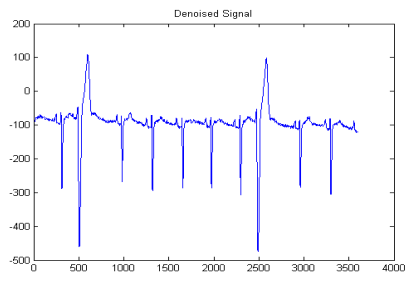

(b)

Fig. 2. (a) Noisy ECG Signal (MIT BIH record 119e24), (b) Denoised ECG Signal using proposed approach

It has been experimentally analyzed that the biorthogonal wavelets appears most promising among all as it gives high quality results on all the signals evaluated by the proposed algorithm. Higher value of SNR obtained by biorthogonal wavelets depicts better noise suppression in comparison to other wavelet families. The application of morphology with wavelets overcomes the need of wavelet thresholding.

\section{Conclusion}

This paper presents the decomposition of the ECG signal using wavelets followed by noise filtering through morphology. The ECG signal decomposition helps in multiscale analysis which provides noise filtering only in the corrupted sub-bands. Noise suppression is achieved through morphological filtering using linear structuring elements. This enables proper removal of residual impulse noise by applying morphological filter only in the affected sub-bands without introducing any distortion in ECG waveform. The proposed algorithm provides good results by bi-orthogonal wavelet in comparison to other wavelet families in terms of SNR values.

\section{References}

1. Dupre, A., Vincent, S., Iaizzo, P.A.: Basic ECG Theory, Recordings and Interpretation. In: Handbook of Cardiac Anatomy, Physiology, and Devices, pp. 191-201 (2005) 
2. Gupta, R., Bera, J.N., Mitra, M.: Development of An Embedded System and MATLABBased GUI for Online Acquisition and Analysis of ECG Signal. Journal Measurement 43(9), 1119-1126 (2010)

3. Jain, A., Singh, S., Bhateja, V.: A Robust Approach for Denoising and Enhancement of Mammographic Breast Masses. International Journal on Convergence Computing 1(1), 38-49 (2013)

4. Bhateja, V., Urooj, S., Pandey, A., Misra, M., Lay-Ekuakille, A.: A Polynomial Filtering Model for Enhancement of Mammogram Lesions. In: Proc. of IEEE International Symposium on Medical Measurements and Applications (MeMeA 2013), Gatineau, Quebec, Canada, pp. 97-100 (2013)

5. Bhateja, V., Urooj, S., Misra, M., Lay-Ekuakille, A.: A Robust Polynomial Filtering Framework for Mammographic Image Enhancement from Biomedical Sensors. IEEE Sensors Journal 13(11), 4147-4156 (2013)

6. Bhateja, V., Urooj, S., Pandey, A., Misra, M., Lay-Ekuakille, A.: Improvement of Masses Detection in Digital Mammograms employing Non-Linear Filtering. In: Proc. of IEEE International Multi-Conference on Automation, Computing, Control, Communication and Compressed Sensing (iMac4s 2013), pp. 406-408 (March 2013)

7. Srivastava, A., Alankrita, R.A., Bhateja, V.: Combination of Wavelet Transform and Morphological Filtering for Enhancement of Magnetic Resonance Images. In: Snasel, V., Platos, J., El-Qawasmeh, E. (eds.) ICDIPC 2011, Part I. Communications in Computer and Information Science, vol. 188, pp. 460-474. Springer, Heidelberg (2011)

8. Raj, A., Alankrita, A.S., Bhateja, V.: Computer Aided Detection of Brain Tumor in Magnetic Resonance Images. International Journal of Engineering and Technology (IACSIT) 3(5), 523-532 (2011)

9. Urooj, S., Lay-Ekuakille, A., Ansari, A.Q., Khan, M., Vergallo, P., Trotta, A.: Volumetric estimation of thorax with cylindrical model and anthropometric measurements. In: 2011 IEEE International Workshop on Medical Measurements and Applications Proceedings (MeMeA), pp. 213-216. IEEE (May 2011)

10. Urooj, S., Khan, M., Ansari, A.Q.: Thorax Physiological Monitoring and Modeling for Diagnosis of Pulmonary Edema. International Journal of Measurement Technologies and Instrumentation Engineering (IJMTIE) 1(2), 54-60 (2011)

11. Urooj, S., Khan, M., Ansari, A.Q., Salhan, A.K.: Measurement of Thoracic Impedance and Approximations: A Diagnosis Technique for Clinical Utilization. Indian Journal of Industrial and Applied Mathematics 3(2), 85-93 (2012)

12. Urooj, S., Khan, M., Ansari, A.Q., Lay-Ekuakille, A., Salhan, A.K.: Prediction of quantitative intrathoracic fluid volume to diagnose pulmonary oedema using LabVIEW. Computer Methods in Biomechanics and Biomedical Engineering 15(8), 859-864 (2012)

13. Sun, Y., Chan, K.L., Krishnan, S.M.: ECG Signal Conditioning by Morphological Filtering. Computers in Biology and Medicine 32(6), 465-479 (2002)

14. Chu, C.-H.H., Delp, E.J.: Impulsive Noise Suppression and Background Normalization of Electrocardiogram Signal Using Morphological Operators. IEEE Transactions on Biomedical Engineering 36, 262-273 (1996)

15. Liu, Z., Wang, J., Liu, B.: ECG Signal Denoising Based on Morphological Filtering. In: Proc. of the 5th International Conference on Bioinformatics and Biomedical Engineering, Wuhan, pp. 1-4 (May 2011)

16. Sayadi, O., Shamsollahi, M.: Multiadaptive Bionic Wavelet Transform: Application to ECG De-noising and Baseline Wandering Reduction. EURASIP Journal on Advances in Signal Processing 2007, 1-11 (2007) 
17. Alfaouri, M., Daqrouq, K.: ECG Signal De-noising by Wavelet Transform Thresholding. American Journal of Applied Sciences 5(3), 276-281 (2008)

18. Sharma, L.N., Dandapat, S., Mahanta, A.: ECG Signal denoising Using Higher Order Statistics in Wavelet Subbands. Biomedical Signal Processing and Control 5, 214-222 (2010)

19. Verma, R., Mehrotra, R., Bhateja, V.: An Improved Algorithm for Noise Suppression and Baseline Correction of ECG Signals. In: Satapathy, S.C., Udgata, S.K., Biswal, B.N. (eds.) Proc. of Int. Conf. on Front. of Intell. Comput. AISC, vol. 199, pp. 733-739. Springer, Heidelberg (2013)

20. Verma, R., Mehrotra, R., Bhateja, V.: A New Morphological Filtering Algorithm for PreProcessing of Electrocardiographic Signals. In: Proc. (SPRINGER) of the Fourth International Conference on Signal and Image Processing (ICSIP 2012), Coimbatore, India, pp. 193-201 (December 2012)

21. Mehrotra, R., Verma, R., Bhateja, V.: An Integration of Improved Median and Morphological Filtering Techniques for ECG Processing. In: Proc. of the 3rd International Advance Computing Conference (IACC 2013), Ghaziabad, U.P., India, pp. 1212-1217 (2013)

22. Bhateja, V., Devi, S.: A Novel Framework for Edge Detection of Microcalcifications using a Non-Linear Enhancement Operator and Morphological Filter. In: Proc. of IEEE 3rd International Conference on Electronics \& Computer Technology (ICECT 2011), Kanyakumari, India, vol. 5, pp. 419-424 (April 2011)

23. Lay-Ekuakille, A., Vergallo, P., Griffo, G., Conversano, F., Casciaro, S., Urooj, S., Bhateja, V., Trabacca, A.: Mutidimensional Analysis of EEG Features using Advanced Spectral Estimates for Diagnosis Accuracy. In: Proc. of IEEE International Symposium on Medical Measurements and Applications (MeMeA 2013), Gatineau, Quebec, Canada, pp. 237-240 (May 2013)

24. Moody, G.B., Mark, R.G.: Impact of the MIT-BIH Arrhythmia Database. IEEE Engineering in Medical and Biology Magazine 20, 45-50 (2001)

25. Jain, A., Bhateja, V.: A Novel Image Denoising Algorithm for Suppressing Mixture of Speckle and Impulse Noise in Spatial Domain. In: Proc. of IEEE 3rd International Conference on Electronics and Computer Technology (ICECT), Kanyakumari, India, vol. 3, pp. 207-211 (2013)

26. Jain, A., Bhateja, V.: A Full-Reference Image Quality Metric for Objective Evaluation in Spatial Domain. In: Proc. of IEEE International Conference on Communication and Industrial Application (ICCIA), Kolkata, W. B., India, pp. 91-95 (2011)

27. Singh, S., Jain, A., Bhateja, V.: A Comparative Evaluation of Various Despeckling Algorithms for Medical Images. In: Proc. of (ACMICPS) CUBE International Information Technology Conference \& Exhibition, Pune, India, pp. 32-37 (2012) 